\title{
The Design of a Hospital Environment Data Acquisition System Based on ARM11 and Embedded Linux
}

\author{
Hong Shao, Chen Yang, Na Zong, Ke-feng Jin \\ School of Information Science and Engineering \\ Shenyang University of Technology \\ Shenyang, China \\ shaoh1974@hotmail.com
}

\begin{abstract}
This paper introduces the current world popular embedded Linux operating system, the latest ARM series chip and several digital sensors, such as temperature, humidity, light and so on, which are applied in the data acquisition terminal of the hospital environment intelligent monitoring system and used to monitor the environmental parameters of the hospital, such as temperature, humidity and light. Based on the embedded Linux operation system's good portability and perfect support on I2C bus and GPIO and the ARM11 processor's high efficiency and low consumption performance, a environment acquisition terminal is designed, which possess strong compatibility and low power consumption, and run well in practice.
\end{abstract}

Keywords-data acquisition; ARM11; Linux kernel module; I2C; GPIO; environment sensor

\section{INTRODUCTION}

Hospital environment parameter, such as temperature, humidity and light, is the hot spot in the field of hospital monitoring. The monitoring of hospital environment situation can be achieved with the hospital environment parameter being monitored strictly, and finally keep people healthy. Currently most monitoring systems are controlled by MCU (Micro Controller Unit) and the numbers and types of environment parameter, which are monitored, are relatively deficient. Simultaneously, the computing speed, storage space and processing capability of MCU are limited. And a relatively large program or a operating system with real-time and multi-tasking capabilities cannot be run on a MCU. Therefore, the development of environment monitoring systems encounters the tremendous bottleneck [1].

Along with the emergence and development of the embedded Linux, ARM chips and digital sensors, the function of monitoring system is becoming more and more powerful. In this paper, an environment data acquisition system based on the embedded Linux operating system, ARM11 chip and digital sensors is designed. The system is integrated with temperature sensor, humidity sensor and light sensor. The system collects a variety of environment data, which is transferred and displayed on the LCD monitor in real time, achieving the real-time monitoring of a variety of environmental data.

\section{THE OVERALL STRUCTURE OF ACQUISITION SYSTEM}

The structure of system can be divided into the hardware part and the software part. The hardware part mainly includes the ARM11 development board and the data acquisition board which is integrated temperature sensor, humidity sensor and light sensor [2]. The structure of hardware is shown in Fig. 1.

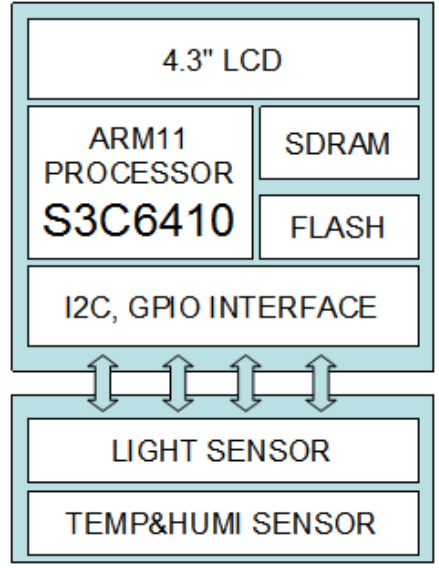

Figure 1. The hardware structure

The software part includes boot-loader, embedded Linux operating system, the Linux kernel driver, the device driver, Qt user interface, etc. The structure of software is given in Fig. 2.

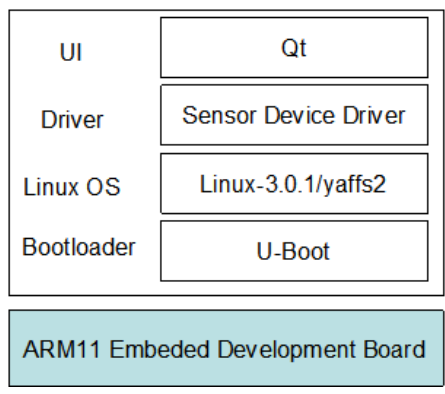

Figure 2. The software structure 


\section{THE HARDWARE DESIGN SCHEME}

Depending on the function of each part, the hardware structure can be divided into two parts which are ARM11 development board and data acquisition board. The ARM development board is used to deal with data processing and display. The data acquisition board is used to deal with data acquisition.

\section{A. ARM11 Development Board}

ARM11 development board is the key part of hardware, which mainly includes master controller, SDRAM, flash, LCD screen, critical peripheral circuits and abundant extended Interfaces [3]. The S3C6410 processor is used as the master controller, which is based on ARM1176JZF-S kernel produced by Samsung Corporation. K9GAG08U0D is chosen as 8bit-2GB Nand Flash chip. And 256MB SDRAM is composed by two K4X1G163PC chips. The 4.3'" TFTLCD touch-screen of WXCAT43-TG3\#001 model is used as LCD screen. The S3C6410 processor possess a wide range of working temperature and humidity, good industrial nature, plenty of external devices, so it is well suitable for a variety of applications in the hospital environment monitoring field, especially in the relatively rugged environment. Therefore, it is chosen as master controller [4]. And the ARM11 development board can work well, after using those high efficient processor and mass storage device.

\section{B. Data Acquisition Board}

In the data acquisition board, the environmental parameters can be collected by sensors and transferred to the processor on ARM11 development board through the interface of the ARM11 development. The data acquisition board and ARM11 development board is connected through DuPont line. Data acquisition board mainly includes the following sections:

1) Light Sensor

The integrated circuit module, which is based on BH1750FVI chip, is adopted as light sensor. The module is an digital Ambient Light Sensor integrated circuit, and it has the following characteristics:

- $\quad$ I2C bus interface (f/s mode Support).

- Two optional I2C_slave address.

- $\quad$ Illuminate to digital converter

- $\quad 1.8 \mathrm{~V}$ logic input interface

DVI is a pin of BH1750FVI chip, and is I2C bus reference voltage terminal. And it is also asynchronous reset terminal. It is necessary to set to ' $\mathrm{L}$ ' after VCC is supplied. In DVI ' $\mathrm{L}$ ' term, internal state is set to Power Down mode. ADDR, SDA, SCL is not stable if DVI ' $\mathrm{L}$ ' term (1us) is not given by systems. In that case, connect the resisters (approximately 100k) to ADDR without directly connecting to VCC or GND, because it is 3 state buffers for internal testing [6].

The chip can be used to achieve direct output of the digital luminance value. Table 1 explains circuit module pins of integrated BH1750FVI chip.
TABLE I. LIGHT SENSOR PIN DESCRIPTION

\begin{tabular}{|l|l|l|}
\hline \multicolumn{1}{|c|}{ Pin } & \multicolumn{1}{|c|}{ Name } & \multicolumn{1}{c|}{ Comment } \\
\hline 1 & GND & Ground \\
\hline 2 & ADD & Address select \\
\hline 3 & SDA & Serial data bidirectional \\
\hline 4 & SCL & Serial clock input \\
\hline 5 & VCC & Supply 2.7-5.5V \\
\hline
\end{tabular}

\section{2) Temperature and Humidity Sensor}

DHT90 digital sensor module is adopted as temperature and humidity sensor. The module not only has fast response and strong anti-interference, but also can be directly accessed by any controller.

DHT90 module is based on SHT10 chip, which have the calibrated digital signal output. DHT90 module pins are indicated in Table 2 [5].

TABLE II. DHT90 PIN DESCRIPTION

\begin{tabular}{|l|l|l|}
\hline Pin & Name & \multicolumn{1}{|c|}{ Comment } \\
\hline 1 & GND & Ground \\
\hline 2 & DATA & Serial data bidirectional \\
\hline 3 & SCK & Serial clock input \\
\hline 4 & VDD & Supply 2.4-5.5V \\
\hline
\end{tabular}

Supply voltage of the DHT90 is $2.4-5.5 \mathrm{~V}$, and the recommended supply voltage is $3.3 \mathrm{~V}$. A $100 \mathrm{nF}$ capacitor should be added between the power supply pin VDD and GND for decoupling filtering. The design of DHT90 peripheral circuit is shown in Fig. 3.

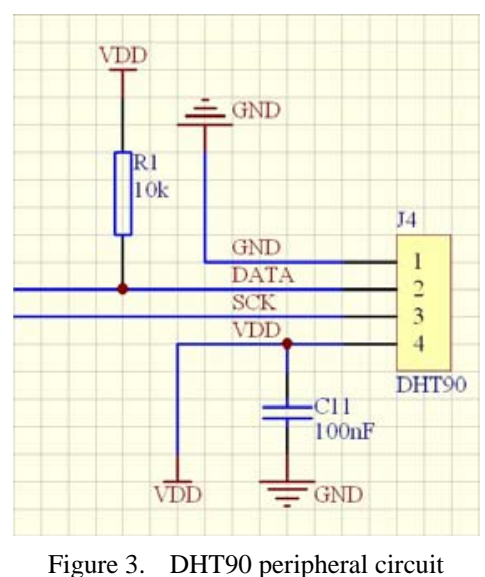

The sensor signal and power consumption of the DHT90 is optimized. DHT90 is not compatible with I2C interface. But DHT90 can be mounted to I2C interface, only if any devices have not been mounted to it. And DHT90 must be operated under its communication timing.

These sensors are concentrated together in a circuit board and connected with ARM11 development boards through DuPont lines. And finally, a data acquisition terminal is grouped with these devices. The reasonable planning of 
module layout and miniaturization of the module can be achieved through this, which can adapt the special and complex hospital environment.

\section{SOFTWARE DESIGN SCHEME}

The embedded Linux is applied in the software design scheme, which include Uboot(v1.8), Linux kernel(v3.0.1), yaffs2, Qt, device driver etc. In this paper, the design of hardware device driver program is mainly introduced, as the transplantation process of the embedded Linux system has been well known. The system can work normally only if the acquisition device worked with the highly efficient and flexible device driver program [7].

\section{A. Design of Light Sensor Driver}

Light sensor is supported with the I2C bus interface. The control method to light sensor is designed based on the structure of I2C bus in embedded Linux [8].

The I2C device is supported perfectly by the Linux system. In the Linux system, the I2C driver program can be divided logically into three parts: the I2C bus driver (I2C_core), the I2C controller (driver I2C_adapter) and the I2C equipment driver (I2C_driver) [9]. The structure of the I2C bus in embedded Linux is shown in Fig. 4.

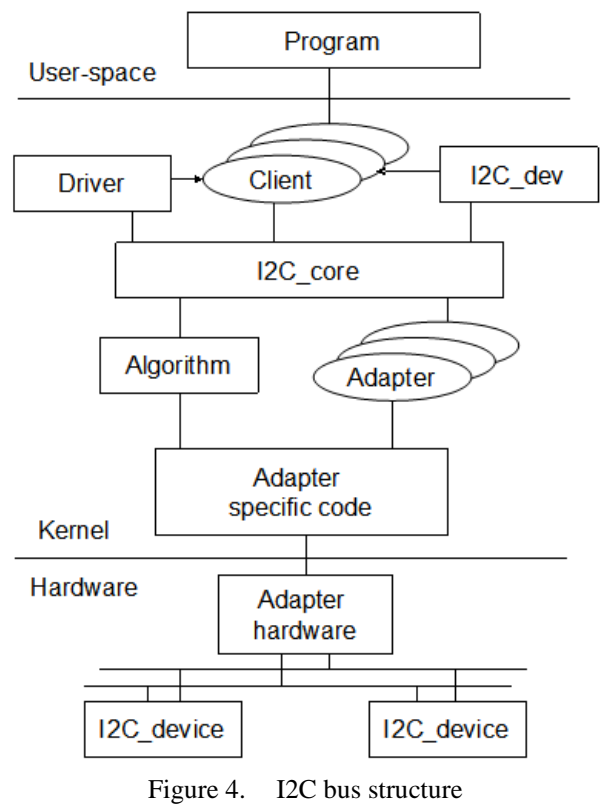

The interface of specific I2C device driver is achieved from two aspects, one is the I2C_core layer interface, which is used to mount I2C_adapter layer to realize the I2C bus and the access method to specific I2C device, including to realize interface function of attach_adapter(), detach_client(), command(). The other one is the user application layer interface, which provide the interface used by user application program to access the I2C device, including realize the standard file operation interface function, such as open, release, read, write and the most important ioctl.
A data structure of struct i2c_driver must be realized, and it registers an I2C driver to I2C_core in the initialization of drive module, and completes the related operation of I2C_adapter. The realization of struct i2c_driver is shown below.

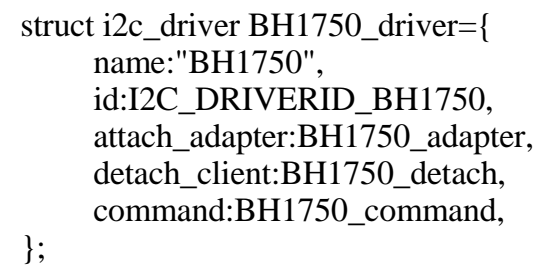

The i2c_probe () provided by I2C_core layer is called to find the $\mathrm{I} 2 \mathrm{C}$ bus and check if there is any $\mathrm{BH} 1750$ device exist in the I2C bus. If there is a BH1750, then the BH1750 device and the corresponding I2C_adapter will be mounted together. The BH1750 can be accessed through the I2C_adapter. At the same time BH1750 is enabled. The i2c_attach_client () is called to register the BH1750 device in I2C_core layer. The i2c_detach_client () is called to released the $\mathrm{BH} 1750$ device in I2C_core layer, then the BH1750 is disabled, the BH1750 is unable to access through I2C driver.

Finally, according to the characteristics of BH1750, A series function, such as BH1750_RWDR, BH1750_READ, BH1750_WRITE, is realized, which is the bottom realization of the ioctl function in user interface.

At the same time, a data structure of struct file_operations must be realized, and a character types of device or a miscdevice device is registered to the kernel.

static const struct file_operations BH1750_fops $=\{$

$$
\begin{array}{lll}
\text {.owner } & =\text { THIS_MODULE, } \\
\text {.llseek } & & =\text { no_llseek, } \\
\text {.read } & & =\text { BH1750_read, } \\
\text {.write } & & =\text { BH1750_write, } \\
\text {.unlocked_ioctl } & =\text { BH1750_ioctl, } \\
\text {.open } & & =\text { BH1750_open, } \\
\text {.release } & & =\text { BH1750_release, }
\end{array}
$$

\}

In the initialization function BH1750 init0 of BH1750 drive module, a I2C driver is registered to I2C_core layer through the i2c_add_driver(\&BH1750_drive) and then BH1750 is registered as a miscdevice device through the misc_register(\&BH1750_miscdev), so that the BH1750 can be accessed by user application program through the device node /dev/BH1750.

Then the BH1750 driver program is compiled into a kernel module. The module is loaded into the kernel through the insmod command, and is tested by user application program [10]. The desired result of all functions realized in BH1750 drive program is achieved, and the light of environment is successfully monitored in the real-time.

\section{B. Design of Temperature and Humidity Sensor Driver}

In this paper, as the I2C bus of ARM11 development board has been occupied by BH1750, the GPIO simulation of I2C bus protocol is carried to control the DHT90 sensor.

GPIO module is also supported well in embedded Linux. In the Linux - 3.0.1 \arch \arm \plat - samsung \include \ plat directory, the head file of gpio-cfg.h can be found. In 
this head file, the following function can be used to control the GPIO port.

int s3c_gpio_cfgpin(unsigned int pin, unsigned int to);

/*set state of GPIO port*/

unsigned s3c_gpio_getcfg(unsigned int pin);

/*get state of GPIO port*/

static inline void gpio_set_value(unsigned gpio, int value);

/*set level of GPIO port*/

static inline int gpio_get_value(unsigned gpio);

/*get level of GPIO port */

Combined with DHT90 communication timing, DHT90 sensor connected in GPIO port of ARM11 development board can be operated through the functions above, and finally achieve the real-time monitoring of temperature and humidity of environment. Then the DHT90 driver is also programmed into a kernel module, then it is compiled and loaded into the kernel, finally tested by user application program with the command of measurement, reset etc [11]. And DHT90 operate normally. The command of DHT90 sensor is shown in Table 3 .

TABLE III.

DHT90 COMMAND LIST

\begin{tabular}{|l|l|}
\hline \multicolumn{1}{|c|}{ Command } & \multicolumn{1}{c|}{ Code } \\
\hline measure temperature & 00011 \\
\hline measure humidity & 00101 \\
\hline read state register & 00111 \\
\hline write state register & 00110 \\
\hline soft reset & 11110 \\
\hline
\end{tabular}

\section{EVAluation}

The experiment in Fig. 5 has demonstrated that the system performs well based on the work above. With this monitor system , the humidity, temperature and illumination of a hospital can be monitored precisely and real-timely, which is extremely important in keeping the people comfortable and healthy in hospital.

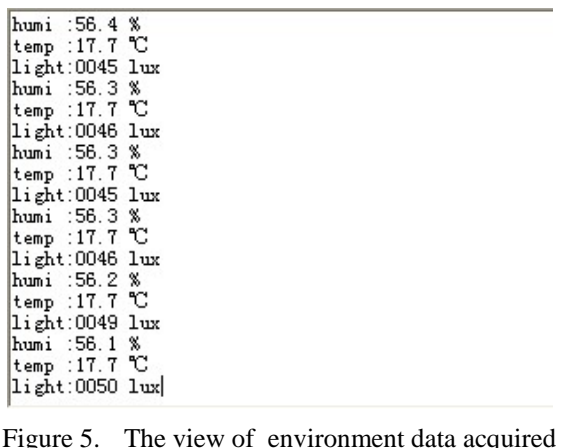

\section{CONCLUSION}

In this paper, based on the ARM11 platform, Linux operating system and digital sensors, an environmental data acquisition terminal is designed. Through development of key technology of software and hardware system, the key circuit and kernel module based on the I2C and GPIO has been designed successfully, the real-time environmental parameters monitoring of temperature, humidity and light has been implemented successfully. The experiment result shows that the structure of whole system is reasonable, which can meet the various demands of environment data acquisition. And the expected aim has been achieved. After further optimization and perfection of the terminal, the terminal will be applied in the hospital environment monitoring widely.

\section{ACKNOWLEDGMENT}

This work is sponsored by the foundation of the Project of Informatization and Information Industry Plan of Shenyang City of China (R201201042) and the Project of College Student Innovation and Entrepreneurship Training Plan of Liaoning Province.

\section{REFERENCES}

[1] L. Xu, "Present Situation and Development of Environmental Monitoring Technology,” Environment Science Survey, vol. 29(z1), pp. 115-118, July 2010.

[2] W. F. Li, X. T. Li, W. J. Luo, J. X. Yang, K. Q. Xu, "A kind of Multifunction Data Processing Terminal Used in Underground Based on ARM11," 2010 International Conference on Multimedia Technology, vol. 5, pp. 42-44, October 2010.

[3] E. Seo, J. Jeong, S. Y. Park, J. Kim, J. Lee, "Catching Two Rabbits: Adaptive Real-time Support For Embedded Linux,” Software, vol. 39(5), pp. 531-550, May 2007.

[4] S. Daud, R. B. Ahmad, N. S. Murthy, "The effects of compiler optimisations on embedded system power consumption," International Journal of Information and Communication Technology, vol. 2(1-2), pp. 73-82, June 2009.

[5] Sensirion AG. SHT1x Datasheet(REV4.3)[EB/OL]

[6] ROHM Semiconductor. BH1750FVI Datasheet(RevD)[EB/OL]

[7] A. Cohen, G. Battistoni, S. Mark, "FLUKA-LIVE-an embedded framework, for enabling a computer to execute FLUKA under the control of a Linux OS,” Radiation Measurements, vol.43(1), pp. 121124, January 2008.

[8] Linux kernel(REV3.0.1)[EB/OL]

[9] M. Q. Cai, A. D. Men, B. Yang, "Study and Application for Driver of I2C Bus Based on Embedded Linux," Computer \& Digital Engineering, vol. 37(7), pp. 181-187, July 2009.

[10] J. Wang, T. F. Mao, Y. G. Chen, "Design and Implementation of Luminometer System Based on Novel BH1750 IC," Journal of Changshu Institute Technology (Natural Sciences), vol.25(2), pp.117120, February 2011.

[11] X. C. Liu, X. L. Liu, N. L. Zhang, "Development of intelligent hygrometer based on DHT90," Journal of Xi'an Shiyou University(Natural Science Edition), vol.25(3), pp. 89-92, March 2010. 